\title{
Lensing Diagnostics of Halo Substructure
}

\author{
Shude Mao \\ Jodrell Bank Observatory, University of Manchester, Macclesfield, \\ Cheshire SK11 9DL, UK
}

\begin{abstract}
The Cold Dark Matter (CDM) hierarchical structure formation theory predicts substructures in dark matter halos. The number of predicted subhalos seems to exceed the observed number of luminous satellite galaxies. Gravitational lenses can be used to probe luminous or dark substructures. Image positions and flux ratios in broad-band (including radio and optical) and emission lines can all be used to probe substructures on different mass scales. The observed gravitational lenses appear to require a few percent of the mass surface density in substructures within the mass range of $10^{4} M_{\odot}-10^{9} M_{\odot}$. Numerical simulations predict roughly the same mass fraction in substructures within the virialised region. But at typical image positions (a few percent of the virial radius), the predicted surface mass density in substructures appears to be lower than required. Both observations and numerical simulations are somewhat uncertain at present so it is not yet clear whether the discrepancy is severe.
\end{abstract}

\section{Introduction}

The Cold Dark Matter (CDM) structure formation can explain an impressive list of observations, including Cosmic Microwave Background (Spergel et al. 2003) and large scale structures. On small scales, the theory, however, appears to be have some problems with rotation curves in low-surface brightness galaxies (de Blok, Bosma, Navarro, Swaters, this volume, for different points of view) and the excess of low angular momentum material in disk galaxies (e.g., van den Bosch et al. 2001). Another challenged prediction of CDM concerns substructures. They are a natural consequence of hierarchical structure formation. As small structures merge to form larger ones, the dense cores of the small structures may survive tidal disruptions. The presence of substructures has been found in both semi-analytical models (Kauffmann et al. 1993) and numerical simulations (e.g., Klypin et al. 1999; Moore et al. 1999; Ghigna et al. 2000; Springel et al. 2001). The number of predicted subhalos in high-resolution numerical simulations exceeds the observed luminous satellite galaxies. One possible solution is that some of these substructures (particularly the lowest mass ones) are dark, and so we cannot observe them in light. It is possible to constrain these substructures dynamically. Another probe of substructures is gravitational lensing as the gravitational deflection does not depend on whether the lenses are dark or luminous. 

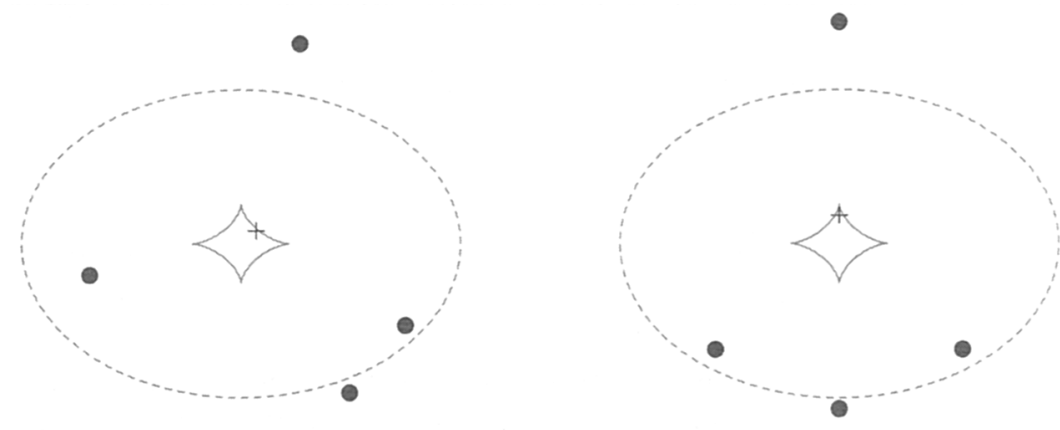

Figure 1. The left panel shows the image configuration for a source close to a fold caustic while the right panel shows that for a source close to a cusp caustic. In both panels, the potential is taken to be a singular isothermal elliptical density distribution with an axis ratio of $q=0.8$. The source position is indicated as a cross while the images are indicated as solid dots. The highly-magnified close pair (bottom right in the left panel) must have approximately equal brightness while for the highly-magnified triple (bottom images in the right panel), the flux of the middle image should be approximately equal to the combined fluxes of the two outer images.

Many papers have been published on this subject (e.g., Mao \& Schneider 1998; Chiba 2002; Evans \& Witt 2003; Bradac et al. 2002; Metcalf \& Madau 2001; Metcalf \& Zhao 2002; Metcalf 2002; Chen et al. 2003). Excellent reviews on this topic can be found in Kochanek \& Dalal (2003a) and Schechter \& Wambsganss (2003). In this article, we more critically examine the claimed agreement between the prediction of CDM and the requirement from gravitational lenses in the literature; the agreement is found to be questionable on a quantitative level. Notice that substructures refer to non-smooth density distributions, so objects ranging from stars to satellite galaxies can all be regarded as substructures. For clarity, we define substructures as those with $M \gtrsim 10^{4} M_{\odot}$, and we refer to the lensing by these objects as milli-lensing in contrast to microlensing by stars. These names are appropriate as their angular Einstein radii are on the order of milli-arcseconds and micro-arcseconds, respectively, in the cosmological context.

\section{Diagnostics for Substructures in Lensing Galaxies}

Gravitational lenses are produced when light from a distant source passes through the gravitational field of an intervening galaxy. If the alignment is sufficiently good, then multiple images of the same source may be formed. The image positions are related to the first order derivatives of the lensing potential through the lens equation, while the image magnifications are related to second-order derivatives. Hence both image positions and flux ratios have sensitivities to the 
potential, including contributions by substructures. As a higher-order derivative, the magnification is more sensitive to small changes in the potential. Flux ratios in gravitational lenses are often difficult to match with isothermal-type models. This is often referred to as the "anomalous" flux ratio problem (e.g., Schechter \& Wambsganss 2003). This problem is "apparent" when we have close pairs and triples in quadrupole lenses (see below).

Gravitational lensing is a mapping between the source plane and image plane. The Jacobian of the mapping may have singularities. These singularities are called critical curves on the image plane and they are mapped into the caustics on the source plane. Fig. 1 shows an example. The asymptotic magnification behaviour when the source is close to a fold or a cusp caustics is well known (see Fig. 1). A close pair must have equal brightness, while for a close triple the flux of the middle image should be equal to the combined fluxes of the two outer images. These relations are valid for any smooth potential, independent of radial and angular profiles. The close pairs and triples are easy to identify in quadrupole systems. The largest well-defined sample from the Cosmic Lens All-Sky Survey (CLASS, Browne et al. 2003; Myers et al. 2003) have 7 simple quadrupole systems. Five of these have close pairs while the other two have close triples. Six out of these seven cases seem to deviate from the expected asymptotic relation. This has been argued as evidence for substructures on the scale of the separations of the images (a few tenths of arcseconds, e.g., Mao \& Schneider 1998; Metcalf \& Zhao 2002). However, one caveat is whether in these cases the lensed sources are sufficiently close to the caustics so that the asymptotic predictions are indeed valid.

Another independent piece of evidence for substructures comes from the fact that saddle images are preferentially dimmed compared to model predictions (Kochanek \& Dalal 2003b). This is expected from milli-lensing by substructures (Keeton 2003) or microlensing by stars (Schechter \& Wambsganss 2002). On the other hand, such a preferential de-magnification of saddle images is difficult to produce in any other propagational effect. Also note that CLASS, being a radio survey, is not affected by differential dust extinction in images.

There are similar astrometric signatures for sources with extended structures. Evidence ranges from bent jets in B1152+199 (Metcalf 2002), unusual VLBI structures for MG2016+112 (Kochanek \& Dalal 2003a; see also Koopmans et al. 2002) and tangential misalignment in B0128+437 (Biggs et al. 2003). Overall, the astrometric evidence for substructures is somewhat less extensive than those for anomalous flux ratios.

\section{Quantitative Requirements for Substructures in Lenses}

It is easy to obtain a rough estimate of the substructures required for an observed flux anomaly. For this, we use the simplest singular isothermal model. In this model, the magnification of an image is given by $\mu=1 /(1-2 \kappa)$, where $\kappa$ is the physical surface density normalized to the critical surface density $\left(\Sigma_{\mathrm{cr}}\right)$. For a lensing galaxy at redshift of 0.5 and a source at redshift $2, \Sigma_{\mathrm{cr}} \approx 2 \times 10^{3} M_{\odot} \mathrm{pc}^{-2}$. For a perturbation in surface density, $\delta \kappa$, the corresponding fractional change in magnification is given by $\delta \mu / \mu=2(1-\kappa) \mu \delta \kappa$. This shows clearly that highly 
magnified images (such as close pairs and triples) are more easily perturbed in their magnifications (Mao \& Schneider 1998).

For a saddle image with magnification $\mu=-5$ (corresponding to $\kappa=0.6$ ), a perturbation with $\delta \kappa=0.02$ (corresponding to $\delta \Sigma \sim 40 M_{\odot} \mathrm{pc}^{-2}$ ) produces a roughly $10 \%$ change in magnification. From the above simple analysis, a perturbation in substructures of $\delta \kappa / \kappa \sim$ a few percent will be sufficient to produce the observed deviations in flux ratios.

Dalal \& Kochanek (2002) have performed a more detailed statistical analysis. They found in order to explain the observed image configurations, the substructure fraction has to be between $0.6 \%-7 \%$ at a $90 \%$ confidence level; the best fit is about $2 \%$ for a systematic flux error of $10 \%$ (see Dalal \& Kochanek 2002 for details). More recently, Metcalf et al. (2003) have applied the method of Moustakas \& Metcalf (2003) to $2237+0305$ and found that at least $4 \%-7 \%$ of the surface density is in substructures within the mass range of $10^{4}-10^{8} M_{\odot}$. This method utilizes the fact that a lens of mass $M$ affects a region with an angular size $\sim \operatorname{mas}\left(M / 10^{6} M_{\odot}\right)^{1 / 2}$, and hence different masses affect the continuum, the narrow and broad emission line regions differently. So by determining the flux ratios in the continuum, infrared, radio, [OIII] and $\mathrm{H} \beta$ emission lines, one can in principle disentangle stellar microlensing and substructure milli-lensing and infer the substructure fraction.

These two studies both appear to require the substructure fraction to be around a few percent, which is more than the observed luminous substructures such as globular clusters or satellite galaxies (Mao \& Schneider 1998; Chiba 2002) can account for. Hence dark substructures seem to be required.

\section{Substructures Predicted in Numerical Simulations}

As we discussed in the introduction, the CDM structure formation model predicts the existence of substructures both from semi-analytical studies and numerical simulations (e.g., Kauffmann et al. 1993; Klypin et al. 1999; Moore et al. 1999; Ghigna et al. 2000). About 5-10\% of the total mass is predicted to be in substructures with a mass spectrum of $n(M) d M \sim M^{-1.8} d M$, seemingly in agreement with the lensing requirement discussed above.

However, this is the total fraction within the virial radius. As substructures passing close to the centers are more easily tidally disrupted, we expect that most substructures are in the outer parts. As typical images pass within $(1-3 \%)$ of the virial radius in lensing galaxies, we expect the projected fraction of surface mass density in substructures to be lower than the global fraction. To check this, we use the collisionless dark matter simulations of Jing \& Suto (2000, 2002). Twelve haloes in cosmological simulations are selected and re-simulated with much higher resolutions. At the end, each halo has about half a million to one million particles within the virial radius. We used 30 random projections to find the average and scatter in the mass fraction in substructures. We find that the typical fraction of surface density in substructures is $<1 \%$ (Mao et al. 2003) at a few percent of the virial radius. These substructures range from $10^{-4}$ to $10^{-1}$ of the parent virial mass (corresponding to $10^{8} M_{\odot}$ to $10^{11} M_{\odot}$ for a $10^{12} M_{\odot}$ halo). We also found that the inclusion of matter outside the virial radius only increases the fraction moderately. 
There are uncertainties in this prediction (see Mao et al. 2003 for more details). To affect the flux ratios efficiently, the subhalo mass distribution needs to be compact, i.e., preferably on the order of the separation of close images $(\sim \mathrm{kpc})$. This may be particularly difficult to satisfy for the subhalos in the outer part. A rough estimate indicates the surface mass density in substructures may be reduced by a factor of $2-5$. The effect of baryon settling toward the center further dilutes the substructure fraction by $\sim 20-50 \%$. To summarise, various effects may reduce the substructure fraction at typical lensing positions to $\lesssim 0.5 \%$ or lower. This seems to be lower than the claimed requirement from gravitational lensing (Dalal \& Kochanek 2002; Metcalf et al. 2003).

\section{Summary and Discussion}

We have reviewed the diagnostics for substructures from gravitational lenses. It appears that in order to reproduce the observed image configurations one requires a few percent of mass surface density in substructures. This is larger than what is seen in CDM. Several effects (including the effect of baryons and the compactness requirement) may reduce the predictions even further, making the match between observations and theories even more difficult to attain.

However, at present both observations and theories are uncertain. The list of cautionary notes is long:

1. The statistical studies by Dalal \& Kochanek (2002) used isothermal plus shear models. It is not clear whether such models are sufficiently realistic and whether slightly more complex models can match the "anomalous" flux ratios (Evans \& Witt 2003; see, however, Kochanek \& Dalal 2003b, for counter arguments). In any case, some of the close pairs may be yet to reach the asymptotic regime.

2. In radio lenses, dust extinction plays no role, but the effect of scattering by free electrons along the line of sight may complicate the interpretation of "anomalous" flux ratios.

3. In numerical simulations, it is not clear what is the best way of defining substructures. It would be interesting to check whether different definitions of substructures yield different results.

4. If the claim by Metcalf et al. (2003) is correct, the required substructure is in the range of $10^{4}-10^{8} M_{\odot}$. The simulations of Jing \& Suto $(2000$, 2002) do not yet reach such low-mass for a typical lensing galaxy. Higher resolution simulations incorporating baryonic effects are required. However these small haloes will be affected by many physical processes (UV background, feedback) that are difficult to model realistically.

Fortunately, much progress can be made from observations that are already planned using the Hubble Space Telescope and the VLBA. These observations will provide more constraints on the lens models especially if the source is extended or if the host galaxies are mapped into arcs or rings. More examples of infrared integral field spectroscopy similar to that performed by Metcalf et al. (2003) would be important. If future observations indicate the discrepancy 
between CDM and lensing persists, then more speculative scenarios such as massive black holes $\left(M \sim 10^{5}-10^{6} M_{\odot}\right)$ in the halo may provide viable alternatives (Lacey \& Ostriker 1985; Xu \& Ostriker 1994).

Acknowledgments. I thank Y.P. Jing, J.P. Ostriker, and J. Weller for collaborations on the work in $\S 4$. I acknowledge useful discussions with I. Browne, H. J. Mo and S. White. I also thank Mark Walker for his hospitality and the symposium organizers for an enjoyable meeting.

\section{References}

Biggs, A. et al. 2003, MNRAS, submitted

Bradac, M., et al. 2002 A\&A, 388, 373

Browne, I. W. A., et al. 2003, MNRAS, 341, 13

Chen, J., Kravtsov, A. V., \& Keeton, C. R. 2003, preprint (astro-ph/0302005)

Chiba M. 2002, ApJ, 565, 17

Dalal, N., \& Kochanek, C. S. 2002, ApJ, 572, 25

Evans, N. W., \& Witt, H. J. 2003, MNRAS, 345, 1351

Ghigna, S., et al. 2000, ApJ, 544, 616

Jing, Y. P., \& Suto Y. 2000, ApJ, 529, L69

Jing, Y. P., \& Suto Y. 2002, ApJ, 574, 538

Kauffmann, G., White, S. D. M., \& Guiderdoni, B. 1993, MNRAS, 264, 201

Keeton, C. R. 2003, ApJ, 584, 664

Klypin, A., Kravtsov A. V., \& Valenzuela O. 1999, ApJ, 522, 82

Kochanek, C. S., \& Dalal, N. 2003a, AIP Conf. Proc. 666, 103

Kochanek, C. S., \& Dalal, N. 2003b, preprint (astro-ph/0302036)

Koopmans, L. V. E., et al. 2002, MNRAS, 334, 39

Lacey, C. G., \& Ostriker, J. P. 1985, ApJ, 299, 633

Mao, S., \& Schneider, P. 1998, MNRAS, 295, 587

Mao, S., Jing, Y. P., J. P. Ostriker, \& Weller, J. 2003, ApJ, submitted

Metcalf, R. B. 2002, 580, 696

Metcalf, R. B., \& Madau, P. 2001, ApJ, 563, 9

Metcalf, R. B., \& Zhao, H. S. 2002, ApJ, 567, L5

Metcalf, R. B., et al. 2003, astro-ph/0309738

Moore, B., Ghigna, S., \& Governato, F. et al. 1999, ApJ, 524, L19

Moustakas, L. A., \& Metcalf R. B. 2003, MNRAS, 339, 607

Myers, S. T., et al. 2003, MNRAS, 341, 1

Schechter, P. L., \& Wambsganss J. 2002, ApJ, 580, 685

Schechter, P. L., \& Wambsganss J. 2003, astro-ph/0309163

Spergel, D. N. S., et al. 2003, ApJS, 148, 175

Springel, V., et al. 2001, MNRAS, 328, 726

van den Bosch, F. C., Burkert, A., \& Swaters, R. A. 2001, MNRAS, 326, 1205

Xu, G., \& Ostriker, J. P. 1994, ApJ, 437, 184 